\title{
Visual rehabilitation of patients with corneal diseases
}

\author{
Michael Oeverhaus ${ }^{1 *}$ D, Dirk Dekowski ${ }^{1}$, Herbert Hirche ${ }^{2}$, Joachim Esser ${ }^{1}$, Barbara Schaperdoth-Gerlings ${ }^{1}$ and \\ Anja Eckstein ${ }^{1}$
}

\begin{abstract}
Background: Although most patients with visual impairment due to corneal diseases can be treated successfully with surgery, some require visual rehabilitation to restore reading ability. To evaluate the best LVAs especially in terms of reading speed and characterize this specific patient group we performed a prospective, randomized crossover trial.

Methods: All 34 patients underwent a detailed examination (slit-lamp, funduscopy, SD-OCT, ETDRS) as screening. Only patients with corneal diseases without other ocular diseases were included. Reading-speed was assessed with International-Reading-Speed-Texts (IReST) consecutively with five different LVAs (low vision aids) during one day in a randomized cross-over design. Corneal haze was quantified with corneal densitometry (Pentacam).

Results: Patients were either visually impaired $(n=28)$, severely impaired $(n=4)$ or legally blind $(n=2)$. Patients read significantly faster with LVAs $(p<0.0001)$. Fastest reading speed could be achieved with video magnifier (CCTV). Optical magnifier and portable-electronic magnifier enabled significantly lower reading speeds $(p<0.01)$. In a subgroup of patients $(\mathrm{VA}<3 / 60, n=6)$ black background enabled patients to read significantly faster compared to white background ( $p=0.03$ ).

Conclusion: Patients with low magnification requirement can be treated successfully with optical LVAs and portable-electronic magnifiers. More severely afflicted patients need a CCTV. Black background enables fastest reading-speeds, probably due to less blinding. Visual impairment can be estimated with corneal densitometry. Our trial confirms the capability of LVAs to successfully restore the reading ability in patients with corneal diseases, which is a crucial part of visual rehabilitation.
\end{abstract}

Trial registration: This trial was registered at the German Clinical Trials Register as DRKS00010887 at 09.08.2016.

Keywords: Low vision, Low vision aids, LVAs, Magnifier, Corneal diseases, Corneal haze, Corneal densitometry

\section{Background}

Visual impairment (VI) is one of the most challenging disabilities worldwide. An increasing number of people are at risk for VI caused by chronic eye diseases due to the globally growing elderly population [1]. Therefore, the requirement for visual rehabilitation is estimated to

\footnotetext{
* Correspondence: michael.oeverhaus@uk-essen.de

'Department of Ophthalmology, University Hospital Essen, Hufelandstr. 55, 45147 Essen, Germany

Full list of author information is available at the end of the article
}

increase in the near future [2]. In Germany, there are estimated to be 1.1 million visual impaired persons (Visual acuity, VA < 6/18), in addition to 160,000 legally blind people (WHO Grade 4, VA $\leq 1 / 60$ ) [3, 4]. The most common causes for VI in Germany are age-related macular degeneration (AMD), glaucoma and diabetic retinopathy [2]. A smaller group of patients suffers from VI due to corneal diseases, like corneal opacities caused by thermal/chemical burns, corneal dystrophies and ocular graft versus host disease (GvHD), as well as

(c) The Author(s). 2020 Open Access This article is licensed under a Creative Commons Attribution 4.0 International License, which permits use, sharing, adaptation, distribution and reproduction in any medium or format, as long as you give appropriate credit to the original author(s) and the source, provide a link to the Creative Commons licence, and indicate if changes were made. The images or other third party material in this article are included in the article's Creative Commons licence, unless indicated otherwise in a credit line to the material. If material is not included in the article's Creative Commons licence and your intended use is not permitted by statutory regulation or exceeds the permitted use, you will need to obtain permission directly from the copyright holder. To view a copy of this licence, visit http://creativecommons.org/licenses/by/4.0/. The Creative Commons Public Domain Dedication waiver (http://creativecommons.org/publicdomain/zero/1.0/) applies to the data made available in this article, unless otherwise stated in a credit line to the data. 
keratoconus. Most cases can be treated successfully with surgical methods like perforating keratoplasty (PK), descemet membrane endothelial keratoplasty (DMEK), deep anterior lamellar keratoplasty (DALK), amnion membrane transplantation and limbal stem cell transplantation. However, due to ocular risk factors, like vascularization, uncontrolled intraocular pressure (IOP) and uveitis some patients cannot be treated successfully with these procedures [5]. In addition, some patients cannot undergo surgery due to comorbidities, like heart diseases or refuse surgery as a result of their age or fear. Even patients who can be treated with surgery often wait months to years for a graft, depending on the procedure. Patients with keratoconus can usually be treated with contact lenses, but some patients suffer from pain while wearing and cannot endure this treatment. Besides these reasons, insufficient health insurance can also keep patients from optimal medical care. All these patients have to endure VI and its consequences, which comprise, besides reading disability, problems in performing tasks of daily living and social interactions. These problems lead to a decreased self-sufficiency and more dependency on relatives and caring persons. Due to these detrimental consequences several studies were able to show, that the quality of life of visual impaired patients is drastically decreasing and there is a higher prevalence of depression [6-8]. Furthermore, patients have more accidents and falls, which leads to a higher morbidity and mortality [9]. Patients with VI should hence undergo visual rehabilitation. By making best use of the remaining vision, rehabilitation aims to improve mobility, reading ability, and consequently autonomy of patients. By low vision aids (LVAs), like optical magnifiers, electronic desk video magnifiers (closed-circuit television, CCTV) and portable electronic vision enhancement systems (pEVES) reading ability can be improved or restored and consecutively quality of life as well [10-15]. The adaption of the suitable LVA for each patient is depending on the disease, the magnification requirement, former reading behavior and other individual factors [16, 17]. Furthermore, the best LVA is highly depending on the task it will be used for. Since electronic LVAs are much more expensive compared to optical LVAs, visual rehabilitation should incorporate this as well [18]. Therefore, the visual rehabilitation process is very complex and time consuming [19]. Due to glare sensitivity and other concomitant problems, like dry eye, adaption of LVAs is especially difficult in patients with corneal diseases. Although it is known that the underlying disease is important for visual rehabilitation there is no published data regarding these patients [16]. This probably results from the rareness of irreversible VI due to corneal diseases. Most studies rather focus on the main causes for visual impairment, like AMD $[19,20]$. The best contrast settings have also only been evaluated for retinal diseases [21-23]. To evaluate LVAs, it is a necessity to measure the reading speed of the patients to show that reading performance can be improved and quantify this improvement, according to a Cochrane Review [24]. This can be done by single sentences charts (Radner, MNREAD) or paragraphs, like the International reading speed texts (IReST). We chose IReST to measure reading speed, because it represents leisure reading (books, newspaper) and provides standardized paragraphs matched for linguistic difficulty to assess reading speed in repeated measurements $[10,25,26]$. Apart from that, it is recommend that patients' preference and characteristics should be assessed [24]. Therefore, in this prospective, randomized cross-over trial, we aimed to characterize patients with visual impairment due to corneal diseases and evaluate the best low vision aid for this group in terms of objective reading performance and patient-reported rating.

\section{Methods \\ Study population}

Patients for this trial were recruited from our low vision and corneal diseases referral center between July 2016 and November 2017. Patients meeting the following inclusion and exclusion criteria were included $(n=34)$ : visual impairment $(\mathrm{BCVA}<6 / 18)$ caused by a corneal disease (e.g. corneal dystrophies, thermal/ chemical burn, ocular GVHD, keratoconus), no visual limitation due to other concomitant ophthalmic diseases (e.g. AMD, glaucoma etc.), ability to converse, read and write German fluently, mentally competent, no diagnosed depression, no physical disability that prevents to operate LVAs [27]. The recruitment and research protocols were reviewed and approved by the Institutional ethics commission, and written informed consent was obtained from all study participants in compliance with the Declaration of Helsinki. The trial was registered with the German Clinical Trials Register (DRKS00010887).

\section{Morphological examination}

All patients were subjected to a standardized ophthalmological examination of both eyes including slit-lamp biomicroscopy and funduscopy. Intraocular pressure was assessed with Goldmann Applanation Tonometry (GAT) and the corneal surface examined with fluorescein staining. Furthermore, all subjects underwent slit-lamp photography, laser interference visual acuity measurement (LIVA) and corneal biometry (Pentacam HR, Oculus $\mathrm{GmbH}$, Wetzlar, Germany) of both eyes. To ensure that patients had no concomitant retinal diseases we performed funduscopy and SD-OCT (Spectral-Domain Optical Coherence Tomography [Spectralis HRA + OCT, Heidelberg Engineering, Germany]) in all patients. To 
characterize corneal status, corneal haze was examined quantitatively with the densitometry program of the Pentacam. Here a rotating Scheimpflug camera is combined with a static camera to acquire multiple photographs of the anterior eye segment. We used the 25scans setting in the automatic release mode to minimize examiner-induced errors. A software module enables a standardized corneal densitometry analysis [28, 29]. It measures corneal backscattered light over a 12-mmdiameter area and full corneal thickness. Corneal densitometry can also be measured in four annular zones centered on the apex of the cornea $(0-2,2-6,6-10$, and $10-12 \mathrm{~mm}$ in diameter). The densitometry measurement can also be provided for the anterior layer (first 120 $\mathrm{mm}$ ), central layer (from the first $120 \mathrm{~mm}$ to the posterior $60 \mathrm{~mm}$ ), and the posterior layer of the cornea $(60$ $\mathrm{mm})$. Densitometry is expressed in grayscale units (GSU), ranging from a minimum light scatter of 0 (no corneal haze) to a maximum light scatter of 100 (totally opaque cornea) [29, 30]. Corneal densitometry has previously been established to quantify the manifestation of several corneal diseases and was therefore chosen to characterize this patient group [31-35]. Slit lamp and Pentacam examinations were all performed by one ophthalmologist to secure homogeneity and reproducibility. Only Pentacam images with good quality were included, according to the integrated quality assurance software.

\section{Functional examination}

Following enrollment, patients underwent a visual assessment to describe multiple aspects of visual function. Distance visual acuity was determined in a standardized manner for each eye (right eye first) according to the ETDRS (Early Treatment of Diabetic Retinopathy Study) protocol. All measurements were performed in the same room under the same conditions (dark room). Internally illuminated Bailey-Lovie charts (Lighthouse International, NY, USA) were used to determine the monocular best corrected visual acuity at $2 \mathrm{~m}$ distance. Letter by letter scoring was employed in accordance with the method described by Ferris [36]. Refraction was performed at $2 \mathrm{~m}$ with Chart " $\mathrm{R}$ " according to the EDTRS protocol. Briefly, the most positive or least negative spherical and least negative cylindrical lens consistent with best visual acuity was used. In addition, near VA was assessed binocularly at $40 \mathrm{~cm}$ using Bailey-Lovie charts and best correction for the near distance [37]. The results are presented in logMAR units. According to the WHO definitions patients were categorized into different classes of visual impairment: WHO grade 1 (VA $<6 / 18$ ), WHO Grade 2 (VA $<6 / 60$ ), WHO Grade 3 $(<3 / 60)$ and Blindness (WHO Grade $4, \leq 1 / 60)$. For statistical analysis we further grouped Grade 1-2 as moderate VI and 3-4 as severe VI. The appropriate magnification was assessed as in clinical routine using standardized charts with sentences in different print sizes at $25 \mathrm{~cm}$ distance under standardized illumination with best correction for near distance, described by others before [19, 38]. Briefly, the smallest print size which still enabled fluent reading was chosen as required magnification which indicates by how much newspaper print size has to be magnified. The LVAs already owned and used regularly by the patients were assessed with a questionnaire as well as their glare sensitivity.

\section{Low vision aids}

We used the International Reading Speed Texts (IReST) to assess the reading speed of patients. To be comparable between each other, the paragraphs were originally designed by linguists who matched content, length (paragraphs, on average 132 words), difficulty (reading ages $10-12$ years) and linguistic complexity. All paragraphs are printed in Times New Roman 10 point with interline and letter spacing similar to newspaper print. Reading speed (right words per minute, wpm) was first assessed with best correction following the protocol described prior to this [39]. In short, subjects had to read the text aloud as quickly as possible without corrections. The wrongly read words were counted and subtracted from the total number of words of the paragraph. Time was measured beginning at the uncovering of the text to have an exact starting point. After this baseline reading speed, 5 LVAs were tested using different paragraphs (No. 2,4,5,6,8) adapted to the magnification requirement of the patient: optical magnifier (Schweizer Optik), pEVES (Reinecker Mano M, 4.3" display), CCTV (Reinecker Veo, 22" display) black font on white background (normal contrast), white-on-black (reversed polarity) and green-on-black. The order of testing was randomized by block randomization to minimize confounding factors. Between testing the different LVAs, all patients had to rest to limit exhaustion and apply artificial tears if required. If the patient was not used to any of the devices, a training session was conducted before the reading test, followed by a break. Patients were trained how to handle the device and had to read, using it to familiarize the patient with the LVA. After completing all reading tests, patients were asked to rate the LVAs from 0 to 10 (worst - best). Reading speed was measured as right words per minute and compared to the normative reading rates of each text, provided by the IReST Study group $[25,26]$. To compare the different LVA, results are presented as percentage of normal reading speed $\left(\frac{w p m}{n w p m}\right)$. Therefore, small differences of normal reading speed between texts are irrelevant in the statistical comparison of the LVAs. This was necessary since IReST offers texts with the same performance level, but not five with identical level 
(max. 4). Still, the maximum difference between the used texts was only nine words. To also enable comparison with other studies reading speed is also presented as right words per minute.

\section{Statistical evaluation}

For metric data, median values and range or the mean and standard deviation $(\mathrm{SD} \pm$ ) were calculated and differences between groups were evaluated with Student's ttest (two-tailed), if D'Agostino-Pearson normality-test showed normal distribution, if not with Mann-WhitneyTest or Wilcoxon signed rank test. Fisher's exact test was used to evaluate group distributions of binary variables. Repeated measures analysis of variance (ANOVA) was performed to examine the effect of LVA type on reading speed. For exploratory investigation of the influence of baseline visual acuity, reading speed was also analyzed for two groups of participants (VA above and below 20/400). Linear regressions have been performed to test the association between functional and morphological parameters. Multiple linear regression has been performed to assess further influential factors. Level of statistical significance was defined two-tailed as $2 \alpha<$ 0.05. $P$-values are given with $\alpha$-adjustment for multiple testing (Bonferroni). All calculations were performed with SPSS (IBM SPSS Statistics, Chicago, IL, USA, Version 22.0.0,) and Graph Pad Prism (Prism 6 for Windows, Software Inc., San Diego, CA, USA, Version 6.01).

\section{Results}

\section{Study population}

Recruitment took place between 1st July 2016 and 31st November 2017. A total of 46 consecutive patients with corneal diseases were recruited by ophthalmologists. Twelve patients were ineligible because of concomitant retinal diseases, resulting in 34 subjects (23-86 years) participating in this clinical trial. A breakdown of demographic characteristics of these participants is presented in Table 1. Most patients were resident with their family or partner (65\%), retired (56\%) and had completed a vocational training (50\%). Visual impairment (WHO grade 1) was most common (82\%), whereas severe impairment (Grade 3) and blindness (Grade 4) were less frequent. Visual characteristics resembled this classification (see Table 2). Most patients suffered from severe glare sensitivity $(65 \%)$.

\section{Ophthalmological examinations}

All 34 patients included in the analysis met the inclusion and exclusion criteria and were visually impaired due to a corneal disease (s. Table 3 for primary diagnosis). SDOCT scans could not be performed in eight patients due to high grade corneal haze. With the help of funduscopy and LIVA it was assured that patients had no
Table 1 Demographic characteristics of participants recruited to the study

\begin{tabular}{|c|c|c|}
\hline & $n$ & \\
\hline Age (years) & 34 & $65.7 \pm 14.2[23,86]$ \\
\hline Females/ Males & $17 / 17$ & $50 \% / 50 \%$ \\
\hline Duration of disease (years) & 34 & $10[1-76]$ \\
\hline \multicolumn{3}{|l|}{ Visual impairment status } \\
\hline Visually impaired $(\leq 20 / 63)$ & 28 & $82.3 \%$ \\
\hline Severely impaired $(\leq 20 / 400)$ & 4 & $11.8 \%$ \\
\hline Blind $(\leq 20 / 1000)$ & 2 & $5.9 \%$ \\
\hline \multicolumn{3}{|l|}{ Residential situation } \\
\hline Alone & 12 & $35.3 \%$ \\
\hline With spouse/ partner/family & 22 & $64.7 \%$ \\
\hline \multicolumn{3}{|l|}{ Employment status } \\
\hline Employed & 9 & $26.5 \%$ \\
\hline Unemployed & 6 & $17.6 \%$ \\
\hline Retired & 19 & $55.9 \%$ \\
\hline \multicolumn{3}{|l|}{ Education and professional training } \\
\hline Secondary school & 6 & $17.6 \%$ \\
\hline Completed vocational training & 17 & $50 \%$ \\
\hline Qualification for university & 8 & $23.5 \%$ \\
\hline University degree & 3 & $8.8 \%$ \\
\hline
\end{tabular}

Unless otherwise stated data are means \pm SD or proportions (\%) or median [range]

concomitant disease which caused visual impairment. Pentacam scans could be performed in 63 eyes. Corneal haze was too dense in 9 patients. In six cases, Pentacam scans had to be dismissed because the quality standards could not be achieved. Thus, 56 eyes (75.7\%) were included in the final analysis. The measurements of all layers for the central annular zone $(0-6 \mathrm{~mm})$ were considered to be most important for visual acuity and hence used for further analysis. A linear regression was performed to test if the quantified corneal haze correlates with visual acuity. The model showed a significant correlation (F1,55 $=11.8 ; p=0.001, r=-0.45$, s. Fig. 1$)$ : A

Table 2 Visual characteristics and symptoms

\begin{tabular}{ll}
\hline BCVA (better eye, logMAR) & $0.70[2-0.4]$ \\
\hline BCVA (other eye, logMAR) & $1.1[n . l-0.6]$ \\
Binocular near VA & $0.63 \pm 0.2$ \\
Corneal haze (GSU) & $35.7 \pm 14$ \\
Magnification need & $5[1.6-30]$ \\
Glare sensitivity (n) & \\
$\quad$ Low & $26.5 \%[8]$ \\
$\quad$ Moderate & $11.8 \%[4]$ \\
Severe & $61.8 \%[20]$ \\
\hline
\end{tabular}

Unless otherwise stated data are means \pm SD or proportions (\%) or median [range] 
Table 3 Primary ocular pathologies among the participants $(n=34)$

\begin{tabular}{ll}
\hline Primary diagnosis & 7 \\
\hline Thermal/chemical burn & 6 \\
Corneal dystrophy & 6 \\
Ulcer & 3 \\
Keratoconus & 3 \\
GVHD & 9 \\
Other & 9
\end{tabular}

higher degree of corneal haze resulted in decreased visual acuity. To explore the unexplained variability, we performed a multiple linear regression and added sequentially age and LIVA. This model, in which corneal haze remained the most important independent variable, could predict a greater percentage of the variability (F2, $55=10.1 ; p<0.0001, r=0.41)$, but showed still a wide range.

\section{Low vision aids}

Most patients were adequately supplied with LVAs (79\%). However, 10 patients had either no $(n=3)$ or insufficient LVAs $(n=7)$. Patients with moderate VI (WHO 1-2) were mostly aided by optical LVAs, whereas patients with severe VI (WHO 3-4) had significantly more often electronic LVAs (see Table 4).

Interestingly, half of the patients used consumer electronics like smartphones and tablets to magnify texts. As main reasons, they reported to use devices that are less stigmatizing and more convenient, since they are carried with anyway (especially smartphones).

Before testing the reading speed, the magnification requirement was assessed in all patients (s. Table 2) and LVAs adapted accordingly. The reading test was performed with five different LVAs in a randomized order to evaluate the LVA with the best reading speed (see

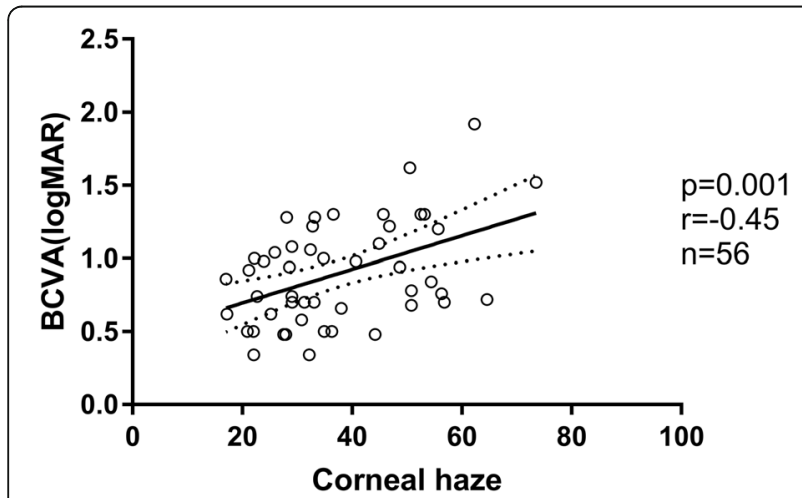

Fig. 1 Linear Regression showed a significant correlation between the degree of corneal haze (0-100 Units, no haze - total opacification) in the central annular zone $(0-6 \mathrm{~mm})$ and best corrected visual acuity (BCVA)
Table 4 Low Vision aids of the patients grouped for grade of visual impairment

\begin{tabular}{|c|c|c|c|}
\hline LVA & $\begin{array}{l}\text { WHO Grade } 1-2 \\
(n=28)\end{array}$ & $\begin{array}{l}\text { WHO Grade 3-4 } \\
(n=6)\end{array}$ & $P$ \\
\hline No LVAs & $11 \%[3]$ & - & $0.93^{\mathrm{a}}$ \\
\hline Insufficient LVAs & $18 \%[5]$ & $33.3 \%[2]$ & $0.21^{\mathrm{a}}$ \\
\hline Optical LVAs & $89 \%[24]$ & $83.3 \%[5]$ & $0.55^{\mathrm{a}}$ \\
\hline Quantity per patient & $1[1,6]$ & $1[0,2]$ & $0.46^{\mathrm{b}}$ \\
\hline Electronic LVAs & $25 \%[6]$ & $88.3 \%[5]$ & $0.01^{\mathrm{a}}$ \\
\hline Quantity per patient & $0[0,3]$ & $1[0,3]$ & $0.04^{b}$ \\
\hline CCTV & $14 \%[4]$ & $66.7 \%[4]$ & $0.02^{\mathrm{a}}$ \\
\hline$p$-EVES & $14 \%[4]$ & $50 \%[3]$ & $0.09^{\mathrm{a}}$ \\
\hline Consumer electronics & $46 \%[12]$ & $83.3 \%[5]$ & $0.18^{\mathrm{a}}$ \\
\hline Quantity per patient & $0[0,4]$ & $1[0,2]$ & $0.34^{\mathrm{b}}$ \\
\hline Smartphone & $46 \%[12]$ & $83.3 \%[5]$ & $0.18^{\mathrm{a}}$ \\
\hline Tablet & $21 \%[40]$ & $50 \%[3]$ & $0.31^{\mathrm{a}}$ \\
\hline Camera & $14 \%[4]$ & - & $0.77^{\mathrm{a}}$ \\
\hline Cut-off filter glasses & $25 \%[6]$ & $16.7 \%[1]$ & $0.66^{\mathrm{a}}$ \\
\hline
\end{tabular}

CCTV Closed-circuit television; $p$-EVES portable electronic vision

enhancement systems

Unless otherwise stated data are proportions (\%) or median [range]

a: Fishers exact test b: Mann-Whitney-test

Table 5 for reading speeds). Upfront, reading was assessed with best correction without any LVA. Only six patients (16\%) could read at intervals of $33 \mathrm{~cm}$, whereas all patients could read with appropriate LVAs. Reading with an optical LVA and p-EVES was impossible in seven and two patients, respectively. Whereas optical magnifier could enable in average fluent reading (80 wpm, [41]) for patients with a max. 5-fold magnification requirement, this was not possible for patients with $\geq 6$ fold. They showed a significantly decreased reading speed $(p<0.0001)$.

Best reading performance for all patients could be achieved with CCTV (see Fig. 2a). Reading speed was significantly higher with CCTV and any contrast compared to optical LVAs and p-EVES (F2,76=24.1; $p<$ 0.001, ANOVA). No significant differences were found

Table 5 Reading speed (right words per minute, wpm) with different LVAs

\begin{tabular}{ll}
\hline Best correction & $21.4 \pm 46$ \\
\hline CCTV & $101.4 \pm 43$ \\
$\quad$ Normal contrast & $102 \pm 36$ \\
Reversed polarity contract & $98.8 \pm 35$ \\
Green-on-black contrast & $69.8 \pm 35$ \\
p-EVES & $65.1 \pm 42$ \\
Optical LVA & \\
\hline $\begin{array}{l}\text { Unless otherwise stated data are means } \pm \text { SD or proportions (\%) or } \\
\text { median [range] } \\
\text { CCTV Closed-circuit television; } p \text {-EVES portable electronic vision } \\
\text { enhancement systems }\end{array}$
\end{tabular}




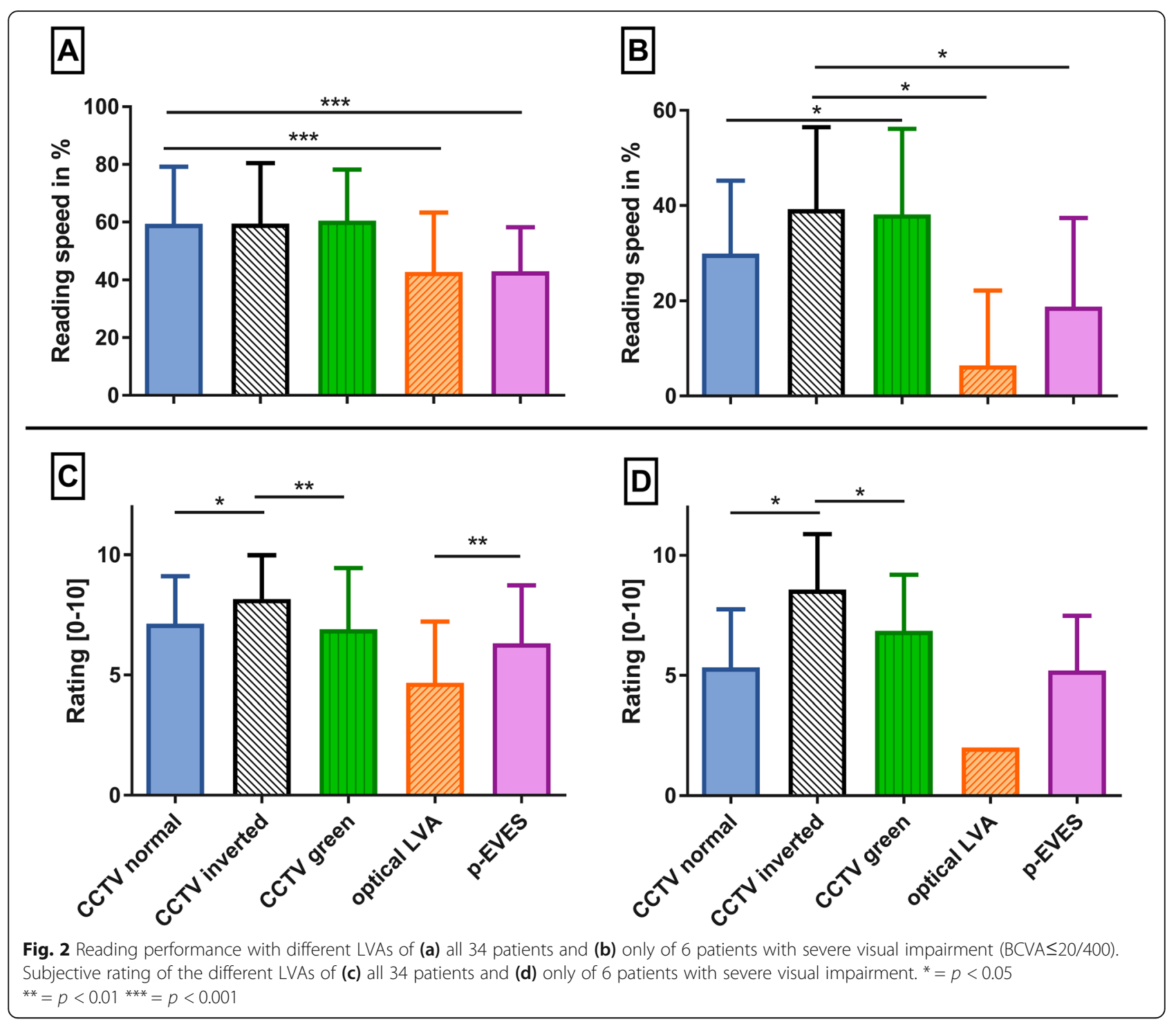

between contrast settings. Normal reading speed (>90\%) could only be achieved by two patients with 2-fold magnification requirement. 'No reading ability' was scored as described before as 0 wpm to compare reading speed with and without LVAs [10]. With LVAs reading speed was significantly increased by $49 \%$ ( $p<0.0001)$. Reading speed was also analyzed with patients allocated to two groups according to their grade of visual impairment (WHO 1-2 vs. WHO 3-4). The first group, comprised only of severely impaired $(n=4)$ and blind patients $(n=$ 2 ), improved their reading speed with LVAs compared to best correction significantly to a lesser extent than the less afflicted group (32\% vs. $59 \%, p=0.04)$. Patients with severe VI showed significant differences in the analysis of varied contrast settings and LVAs (see Fig. 2b): Patients could read significantly faster if the background was black (no significant differences between white or green font color) compared to CCTV with normal contrast $(p=0.03)$ and again compared to optical LVAs and p-EVES $(p<0.001)$. The second group (WHO 3-4, $n=28)$ showed the same results as the analysis of all patients.

The patient-reported rating mostly resembled the results of the reading speed assessment (see Fig. 2c). However, most patients preferred reversed polarity to the normal $(p=0.02)$ and green-on-black contrast $(p=$ 0.006). Interestingly, patients graded p-EVES significantly better than optical LVAs $(p=0.004)$. In the group of severely impaired patients reversed polarity was also rated significantly better compared to normal contrast $(p=0.02)$ with a higher mean difference between both (see Fig. 2d). The green-on-black contrast was rated slightly worse compared to reversed polarity, but better compared to normal contrast (both without significance). In patients with moderate VI there were no significant differences in ratings between the three contrast 
settings. Both portable LVAs were also significantly worse rated compared to CCTV $(p=0.01)$.

To evaluate which functional parameters influence the reading speed we performed linear regressions. The analysis revealed that reading speed is related to near visual acuity $(\mathrm{F} 1,34=24.7 ; p=0.001, r=0.49)$ and magnification requirement $(\mathrm{F} 1,34=23.1 ; p<0.0001, r=-0.51)$. Reading speed decreased with higher magnification and lower near visual acuity (s. Fig. 3a). Age, by contrast, showed no significant correlation (F1,34=0.7; $p=0.46, r=-0.11$ ). To further characterize the patient cohort, we also evaluated the correlation of reading speed to corneal haze. Linear regression showed a significant correlation $(\mathrm{F} 1,34=6.8 ; \mathrm{p}=$ $0.01, r=-0.20$ ). Higher opacification of the cornea lead to lower reading speed (s. Fig. 3b).

\section{Discussion}

In this prospective, randomized cross-over clinical trial a detailed evaluation of patients with visual impairment due to corneal diseases and the best LVAs for them in terms of reading speed and patient-reported rating could be successfully accomplished. Our results show that visual rehabilitation of these specific patients can also be achieved with appropriately chosen LVAs and complement previous reports of treatment of retinal diseases [10, 11]. Whereas only six patients could read without a LVA, all patients could with the help of a LVA. This is similar to findings in AMD and confirms the great value of visual rehabilitation through the careful and adequate provision of LVAs [10]. Since reading is an integral part of many activities, the restoration of reading can also benefit other parts of daily living and consecutively independence and quality of life [11].

\section{Providing the appropriate LVA}

Dependent on the grade of visual impairment, different LVAs could be shown to be effective in increasing the reading speed of the patients. This has previously been shown also for patients with retinal diseases $[10,11]$. Hence, adequate visual rehabilitation needs the appropriate LVA: Severely afflicted patients ( $\geq 6$-fold magnification requirement) could either not read at all with portable LVAs, or only poorly and needed a CCTV to restore their reading ability. In contrast, patients with low magnification requirement could read fluently with optical LVAs. This is in concordance with the visual rehabilitation of general low vision populations $[19,23,38$, 42] and emphasizes again the necessity to assess the magnification requirement before prescribing a LVA to ensure a cost-effective visual rehabilitation. Between both tested handheld LVAs there were no significant differences in reading speed, but patients rated p-EVES significantly better than optical magnifier. This is probably due to the fact that optical magnifier with higher magnification power restrict the field of vision distinctively. Besides that, p-EVES allow handling in habitual distance and better contrast [43]. Despite that, all patients could read fastest with CCTV. This was also the case in a recent trial by Jackson et al. (2017) with AMD patients, as well as in other prior trials with mainly retinal diseased patients $[13,24]$. The superiority of the CCTV over portable LVAs is probably due to the wider field for viewing, which is especially beneficial with higher magnifications, since more words can be seen simultaneously [11, 44, 45]. In general, CCTVs seem ideal for leisure reading, since they offer binocular viewing in a habitual working distance and variable magnification and contrast settings. However, even with CCTVs available, patients' expectations should be managed cautiously, because only two patients could achieve roughly the reading speed of a normal cohort even with CCTV. This has also been shown before for retinal diseases [10, 11]. Patients should thus be informed that LVAs cannot fully
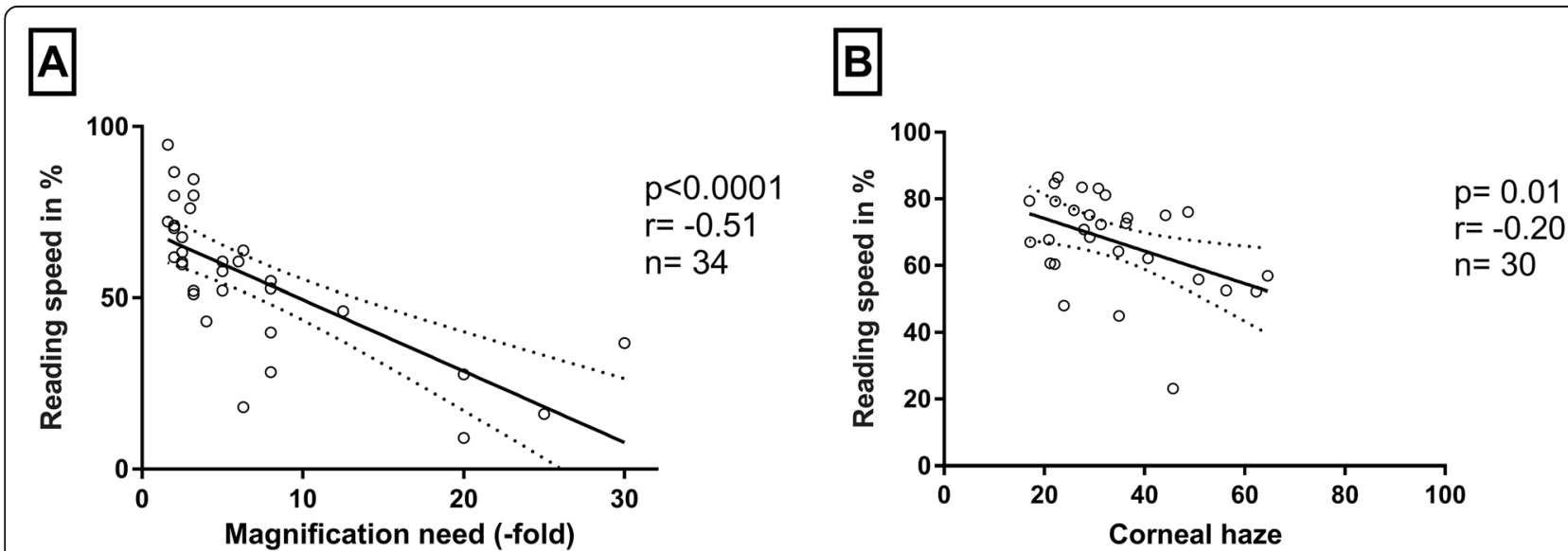

Fig. 3 Reading performance was best predicted by (a) magnification need, but also significantly influenced by (b) the grade of corneal haze (0100 Units, no haze - total opacification) 
compensate a visual impairment in order to ensure their motivation [10]. Furthermore, CCTVs bind patients to a single location, and are more expensive which is why they can only be seen as an addition to a portable LVA for mobile use in shops, banks etc. [46]. Interestingly, already half of the patients used consumer electronics like smartphones as magnifying aid, because they are less stigmatizing than classical LVAs and provide the basic features of a p-EVES [17, 47]. Since many especially young patients own these devices anyway, they should be informed about the possibilities they comprise [17]. To conclude, the LVA for a patient should be chosen according to his magnification requirement, reading performance and personal preference. In principle, visual rehabilitation should aim for an optimal visual and economic outcome. Only after testing the possible LVAs, a patient can decide jointly with the low vision specialist, which device is appropriate for him. A limitation of our trial is that we could not evaluate the long-term use of each device. Future studies should consider this aspect and enable home phases and follow-up visits, since testing in the clinic is an artificial setting and only the daily use enables the patient to really test a LVA [24]. However, the most important task of a LVA is to provide adequate reading assistance, which we evaluated with standardized assessment of reading speed and personal rating.

\section{Contrast setting}

Besides the choice of the right LVA - which we could show, is in patients with corneal diseases also dependent on the magnification requirement - the best contrast setting is of particular importance, since most of these patients suffer severely under glare sensitivity. In the analysis of our whole cohort, there were no differences between the contrast settings in terms of reading speed. This indicates that the most important factor is magnification. For further elucidation, we also analyzed two subgroups stratified according to their visual impairment grade. Whereas patients with moderate VI also showed, as expected, no differences between the contrast settings, this was not the case in patients with severe VI. Here, patients could read significantly faster with a black background compared to normal contrast. Previous studies already established this supremacy of reversed polarity over normal contrast for retinal diseases [22]. However, the color of the font had no significant influence in our cohort, although the mean reading speed was higher with reversed polarity compared to green on black contrast. In contrast, the patient reported rating showed a significant preference of the patients towards reversed polarity compared to normal and green-on-black contrast. This was the case in the analysis of the whole cohort as well. Hence, reversed polarity offers the fastest reading speed and best subjective rating in patients with severe visual impairment. This is in concordance with a previous trial by Legge et al. (1990), who showed that luminance contrast is superior to color contrast and that there is no additive interaction, since both are coded similar in our visual system [48]. When asked, many patients reported that the white background is glaring them. This discomfort is one probable explanation for the decreased reading speed. In less afflicted patients without severe glare, the contrast seems to be less important.

\section{Characterization of the patient group}

Besides the evaluation of the visual rehabilitation, we also aimed to characterize the corneal diseased low vision patients. The reasons for visual impairments were diverse in contrast to regular visually impaired patients, who mostly suffer from AMD [11, 19, 49]. All patients had in common that they could not be treated with surgical procedures anymore and had to endure the consequences of visual impairment consecutively. Interestingly, many patients reported that they have not or only lately been informed about LVAs, since surgical options were still discussed. Even if there are possible options, in the future visual rehabilitation should not be postponed, to prevent a drastic decrease of quality of life and the incidence of depression. For a detailed characterization of these patients, we analyzed the visual impairment in regard to the cornea. For this, we measured the corneal haze with Pentacam. With the quantification of corneal haze and the analysis of its relationship with visual acuity, we could demonstrate that the vision of patients with corneal opacifications depends mainly on the cornea. Previous publications already established the corneal densitometry as a tool to quantify the disease stage [31-35]. In contrast to our results, Kamiya et al. (2017) found no significant correlation between corneal densitometry and visual acuity in patients with band keratopathy, which might be a result of the nature of the specific disease, since the visual axis is not necessarily occluded [50]. For further elucidation of the factors determining the visual acuity of these patients, we performed a multiple regression with corneal haze, age and LIVA. It showed a higher correlation, which indicates that the age-dependent capability of the retina is also important in addition to the grade of corneal haze. Following the decreased visual acuity with higher opacification, reading speed decreased as well with increased corneal haze, as evaluated by linear regression. Mathews et al. (2017) has been able to show before that with higher corneal staining the reading speed decreases [51]. Our results with an objective method confirmed these findings. 


\section{Prediction of performance}

For provision of the appropriate LVA for a visually impaired patient it is important to predict how well the patient will perform with different LVAs [24]. Therefore, we evaluated the reading speed concerning morphological and functional examinations. With linear regression, we could show that reading speed decreases with higher magnification requirement and lower near visual acuity. The influence of visual acuity on reading performance has been shown prior to this for AMD patients [10]. Furthermore, we could show that the objective parameter corneal haze has as well a significant impact on the reading speed. This is in concordance with our finding of decreased visual acuity with worse corneal opacification. However, corneal haze alone is not a valid parameter for precise prediction of VA or reading speed, because of a high variability. Hence, visual rehabilitation for corneal patients can also rely on measurement of the magnification requirement and/or on near visual acuity as in patients with retinal diseases.

\section{Conclusions}

Reading ability in patients with corneal diseases can be restored successfully and improved with LVAs. The appropriate LVA is dependent on the grade of visual impairment, assessed as magnification requirement or near visual acuity. Highest reading speeds could be achieved with CCTVs, especially with black background, probably due to less glare. Severely afflicted patients rated reversed polarity with a CCTV best, less afflicted patients could also improve their reading speed sufficiently with portable LVAs. Quantification of corneal haze can assist to estimate visual acuity and reading performance.

\section{Abbreviations \\ AMD: Age-related macular degeneration; BCVA: Best correct visual acuity; CCTV: Closed circuit television; also electronic desk video magnifiers; DALK: Deep anterior lamellar keratoplasty; DMEK: Descemet membrane endothelial keratoplasty; ETDRS: Early treatment of diabetic retinopathy study; GAT: Goldmann applanation tonometry; GSU: Grayscale units; GVHD: Graft versus host disease; IReST: International-reading-speed-texts; IOP: Intraocular pressure; LIVA: Laser interference visual acuity measurement; logMAR: Logarithmic value of the minimum angle of resolution; LVA: Low vision aid; PK: Perforating keratoplasty; p-EVES: Portable electronic vision enhancement systems; SD-OCT: Spectral-domain optical coherence tomography; VA: Visual acuity; VI: Visual impairment; WHO: World Health Organization; Wpm: Words per minute}

\section{Acknowledgements}

We thank our orthoptist Susanne Walter and our study nurse Ulrike Kaiser for their contribution to this clinical trial.

\section{Authors' contributions}

$\mathrm{MO}$ and $\mathrm{HH}$ analyzed and interpreted the patient data. DD was a major contributor in performing the clinical trial. $A E, B S$ and JE contributed to the study design and interpretation. All authors read and approved the final manuscript.

\section{Funding}

This research was supported by Research grants from QD Laser Inc. QD Laser had no role in the design, conduct, analysis of the clinical trial and the writing of the manuscript.

\section{Availability of data and materials}

The datasets used and analysed during the current study are available from the corresponding author on reasonable request.

\section{Ethics approval and consent to participate}

The recruitment and research protocols were reviewed and approved by the ethics commission of the Medical Faculty of the University Duisburg-Essen (16-6879-BO), and written informed consent was obtained from all study participants in compliance with the Declaration of Helsinki. The trial was registered with the German Clinical Trials Register (DRKS00010887).

\section{Consent for publication}

Not applicable.

\section{Competing interests}

The authors declare that they have no competing interests.

\section{Author details}

${ }^{1}$ Department of Ophthalmology, University Hospital Essen, Hufelandstr. 55, 45147 Essen, Germany. ${ }^{2}$ Institute of Medical Informatics, Biometry and

Epidemiology, University of Duisburg-Essen, Essen, Germany.

Received: 9 November 2018 Accepted: 14 April 2020

Published online: 06 May 2020

\section{References}

1. Bourne RRA, Flaxman SR, Braithwaite T, Cicinelli MV, Das A, Jonas JB, et al. Magnitude, temporal trends, and projections of the global prevalence of blindness and distance and near vision impairment: a systematic review and meta-analysis. Lancet Glob Health. 2017:5(9):e888-e97.

2. Knauer C, Pfeiffer N. Blindness in Germany--today and in 2030. Ophthalmologe. 2006;103(9):735-41.

3. Bertram A. Prevalence and causes for blindness and visual impairment in Germany. Der Augenarzt. 2005;39(6):267-8.

4. Resnikoff S, Pascolini D, Etya'ale D, Kocur I, Pararajasegaram R, Pokharel GP, et al. Global data on visual impairment in the year 2002. Bull World Health Organ. 2004;82(11):844-51.

5. Nguyen NX, Seitz B, Martus $P$, Langenbucher A, Cursiefen C. Long-term topical steroid treatment improves graft survival following normal-risk penetrating keratoplasty. Am J Ophthalmol. 2007;144(2):318-9.

6. Brody BL, Gamst AC, Williams RA, Smith AR, Lau PW, Dolnak D, et al. Depression, visual acuity, comorbidity, and disability associated with agerelated macular degeneration. Ophthalmology. 2001;108(10):1893-900 discussion 900-1.

7. Trousdale ER, Hodge DO, Baratz KH, Maguire LJ, Bourne WM, Patel SV. Vision-related quality of life before and after keratoplasty for Fuchs' endothelial dystrophy. Ophthalmology. 2014;121(11):2147-52.

8. Salive ME, Guralnik J, Glynn RJ, Christen W, Wallace RB, Ostfeld AM. Association of visual impairment with mobility and physical function. J Am Geriatr Soc. 1994:42(3):287-92.

9. Legood R, Scuffham P, Cryer C. Are we blind to injuries in the visually impaired? A review of the literature. Inj Prev. 2002;8(2):155-60.

10. Nguyen NX, Weismann M, Trauzettel-Klosinski S. Improvement of reading speed after providing of low vision aids in patients with age-related macular degeneration. Acta Ophthalmol. 2009;87(8):849-53.

11. Jackson ML, Schoessow KA, Selivanova A, Wallis J. Adding access to a video magnifier to standard vision rehabilitation: initial results on reading performance and well-being from a prospective, randomized study. Digit J Ophthalmol. 2017;23(1):1-10.

12. Hamade N, Hodge WG, Rakibuz-Zaman M, Malvankar-Mehta MS. The effects of low-vision rehabilitation on Reading speed and depression in age related macular degeneration: a meta-analysis. PLoS One. 2016;11(7):e0159254.

13. Burggraaff MC, van Nispen $R M$, Hoeben FP, Knol DL, van Rens $G H$. Randomized controlled trial on the effects of training in the use of closedcircuit television on reading performance. Invest Ophthalmol Vis Sci. 2012; 53(4):2142-50 
14. Burggraaff $M C$, van Nispen $R M$, Knol DL, Ringens PJ, van Rens $G H$. Randomized controlled trial on the effects of CCTV training on quality of life, depression, and adaptation to vision loss. Invest Ophthalmol Vis Sci. 2012;53(7):3645-52.

15. Lamoureux EL, Pallant JF, Pesudovs K, Rees G, Hassell JB, Keeffe JE. The effectiveness of low-vision rehabilitation on participation in daily living and quality of life. Invest Ophthalmol Vis Sci. 2007;48(4):1476-82.

16. Frohlich SJ. Age-related macula degeneration and diabetic retinopathy -differences in optic rehabilitation. Klin Monbl Augenheilkd. 2005;222(4):337-41.

17. Oeverhaus M, Hirche H, Esser J, Eckstein A, Schaperdoth-Gerlings B. Evaluation of the medical treatment situation of the visually impaired: Significant differences between young and old. Ophthalmologe. 2018.

18. Koberlein J, Beifus K, Schaffert C, Finger RP. The economic burden of visual impairment and blindness: a systematic review. BMJ Open. 2013;3(11): e003471.

19. Rohrschneider K, Bruder I, Blankenagel A. Ophthalmological rehabilitation experience at the university eye hospital Heidelberg. Ophthalmologe. 1999; 96(9):611-6.

20. Nguyen NX, Weismann M, Trauzettel-Klosinski S. Spectrum of ophthalmologic and social rehabilitation at the Tubinger low-vision clinic : a retrospective analysis for 1999-2005. Ophthalmologe. 2008;105(6):563-9.

21. Ben Mansour K, Rezzoug N, Jacquier-Bret J, Gorce P. Validation of a low-cost wearable accelerometer for temporal gait parameter quantification. Comput Methods Biomech Biomed Engin. 2014;17(Suppl 1):160-1.

22. Ehrlich D. A comparative study in the use of closed-circuit television reading machines and optical aids by patients with retinitis pigmentosa and maculopathy. Ophthal Physiol Opt : J Br Coll Ophthal Opticians. 1987;7(3):293-302.

23. Kloevekorn-Fischer U, Kloevekorn-Norgall K, Duncker G, GrunauerKloevekorn C. Results of low-vision rehabilitation in vision impaired patients. Klin Monbl Augenheilkd. 2009;226(5):428-31.

24. Virgili G, Acosta R, Grover LL, Bentley SA, Giacomelli G. Reading aids for adults with low vision. Cochrane Database Syst Rev. 2013;10:CD003303.

25. Trauzettel-Klosinski S, Dietz K, Group IRS. Standardized assessment of Reading performance: the new international Reading speed texts IReST. Invest Ophthalmol Vis Sci. 2012;53(9):5452-61.

26. Hahn GA, Penka D, Gehrlich C, Messias A, Weismann M, Hyvarinen L, et al. New standardised texts for assessing reading performance in four European languages. Br J Ophthalmol. 2006:90(4):480-4.

27. $\S 60$ Eingliederungshilfe-Verordnung, §1, (2003).

28. Lopes B, Ramos I, Ambrosio R Jr. Corneal densitometry in keratoconus. Cornea. 2014;33(12):1282-6.

29. Ni Dhubhghaill S, Rozema JJ, Jongenelen S, Ruiz Hidalgo I, Zakaria N, Tassignon MJ. Normative values for corneal densitometry analysis by Scheimpflug optical assessment. Invest Ophthalmol Vis Sci. 2014;55(1):162-8.

30. Cho YK, Chang HS, La TY, Ji D, Kim H, Choi JA, et al. Anterior segment parameters using Pentacam and prediction of corneal endothelial cell loss after cataract surgery. Korean J Ophthalmol. 2010;24(5):284-90.

31. McLaren JW, Wacker K, Kane KM, Patel SV. Measuring corneal haze by using Scheimpflug photography and confocal microscopy. Invest Ophthalmol Vis Sci. 2016;57(1):227-35.

32. Otri AM, Fares U, Al-Aqaba MA, Dua HS. Corneal densitometry as an indicator of corneal health. Ophthalmology. 2012;119(3):501-8.

33. Elflein HM, Hofherr T, Berisha-Ramadani F, Weyer V, Lampe C, Beck M, et al. Measuring corneal clouding in patients suffering from mucopolysaccharidosis with the Pentacam densitometry programme. $\mathrm{Br} J$ Ophthalmol. 2013;97(7):829-33.

34. Malhotra C, Jain AK, Dwivedi S, Chakma P, Rohilla V, Sachdeva K Characteristics of pre-Descemet membrane corneal dystrophy by three different imaging modalities-in vivo confocal microscopy, anterior segment optical coherence tomography, and Scheimpflug corneal densitometry analysis. Cornea. 2015;34(7):829-32.

35. Alnawaiseh M, Rosentreter A, Prokosch V, Eveslage M, Eter N, Zumhagen L. Changes in corneal densitometry in patients with Fuchs endothelial dystrophy after endothelial Keratoplasty. Curr Eye Res. 2017:42(2):163-7.

36. Ferris FL 3rd, Kassoff A, Bresnick GH, Bailey I. New visual acuity charts for clinical research. Am J Ophthalmol. 1982;94(1):91-6.

37. Bailey IL, Lovie JE. The design and use of a new near-vision chart. Am J Optom Physiol Optic. 1980;57(6):378-87.

38. Nguyen N.X. WM, Trauzettel-Klosinski S. Spectrum of ophthalmologic and social rehabilitation at the Tübinger Low-Vision Clinic Ophthalmologe 2008(105):563-569.
39. Altpeter EK, Marx T, Nguyen NX, Naumann A, Trauzettel-Klosinski S. Measurement of reading speed with standardized texts: a comparison of single sentences and paragraphs. Graefe's archive for clinical and experimental ophthalmology = Albrecht von Graefes Archiv fur klinische und experimentelle. Ophthalmol. 2015;253(8):1369-75.

40. Pauklin M, Steuhl KP, Meller D. Limbal stem cell deficiency after chemical burns: investigations on the epithelial phenotype and inflammation status. Ophthalmologe. 2009;106(11):995-8.

41. Whittaker SG, Lovie-Kitchin J. Visual requirements for reading. Optom Vis Sci : Official Publication Am Acad Optom. 1993;70(1):54-65.

42. Rohrschneider K. Optisch und elektronisch vergrößernde Sehhilfen. In: Kampik A, Grehn F, editors. Augenärztliche Rehabilitation. Stuttgart: Thieme Verlag; 2005. p. 35-45.

43. Taylor JJ, Bambrick R, Brand A, Bray N, Dutton M, Harper RA, et al. Effectiveness of portable electronic and optical magnifiers for near vision activities in low vision: a randomised crossover trial. Ophthalmic Physiol Opt : J Br Coll Ophthal Opticians. 2017.

44. Peterson RC, Wolffsohn JS, Rubinstein M, Lowe J. Benefits of electronic vision enhancement systems (EVES) for the visually impaired. Am J Ophthalmol. 2003;136(6):1129-35.

45. Goodrich GL, Kirby J. A comparison of patient reading performance and preference: optical devices, handheld CCTV (Innoventions Magni-cam), or stand-mounted CCTV (Optelec Clearview or TSI genie). Optometry. 2001; 72(8):519-28.

46. Rohrschneider K, Bayer Y, Brill B. Closed-circuit television systems : Current importance and tips on adaptation and prescription. Ophthalmologe. 2017; 115:548-52

47. Crossland MD, Silva RS, Macedo AF. Smartphone, tablet computer and ereader use by people with vision impairment. Ophthal Physiol Opt : J Br Coll Ophthal Opticians. 2014;34(5):552-7.

48. Legge GE, Parish DH, Luebker A, Wurm LH. Psychophysics of reading. XI. Comparing color contrast and luminance contrast. J Opt Soc Am A. 1990; 7(10):2002-10

49. Finger RP. Blindness in Germany: dimensions and perspectives. Ophthalmologe. 2007:104(10):839-44.

50. Kamiya K, Kobashi H, Takahashi M, Shoji N, Shimizu K. Effect of scattering and aberrations on visual acuity for band Keratopathy. Optom Vision Sci : Official Publication Am Acad Optom. 2017;94(11):1009-14.

51. Mathews PM, Ramulu PY, Swenor BS, Utine CA, Rubin GS, Akpek EK. Functional impairment of reading in patients with dry eye. $\mathrm{Br} J$ Ophthalmol. 2017;101(4):481-6.

\section{Publisher's Note}

Springer Nature remains neutral with regard to jurisdictional claims in published maps and institutional affiliations.

Ready to submit your research? Choose BMC and benefit from:

- fast, convenient online submission

- thorough peer review by experienced researchers in your field

- rapid publication on acceptance

- support for research data, including large and complex data types

- gold Open Access which fosters wider collaboration and increased citations

- maximum visibility for your research: over $100 \mathrm{M}$ website views per year

At $\mathrm{BMC}$, research is always in progress.

Learn more biomedcentral.com/submission 